\title{
Practice Variation in Skin Cancer Treatment and Follow-Up Care: A Dutch Claims Database Analysis
}

\author{
Sven van Egmond ${ }^{\mathrm{a}}$ Loes M. Hollestein ${ }^{\mathrm{a}}$ Carin A. Uyl-de Groot ${ }^{\mathrm{b}}$ \\ Judith A. van Erkelens ${ }^{c}$ Marlies Wakkee ${ }^{a}$ Tamar E.C. Nijsten ${ }^{a}$ \\ ${ }^{a}$ Department of Dermatology, Erasmus MC Cancer Institute, Rotterdam, The Netherlands; ${ }^{b}$ Erasmus School of \\ Health Policy and Management, Erasmus University, Rotterdam, The Netherlands; ' $V e k t i s$ B.V., Zeist, \\ The Netherlands
}

\section{Keywords}

Skin neoplasms · Benchmarking · Public health · Treatment · Follow-up

\begin{abstract}
Background: Quality indicators are used to benchmark and subsequently improve quality of healthcare. However, defining good quality indicators and applying them to high-volume care such as skin cancer is not always feasible. Objectives: To determine whether claims data could be used to benchmark high-volume skin cancer care and to assess clinical practice variation. Methods: All skin cancer care-related claims in dermatology in 2016 were extracted from a nationwide claims database (Vektis) in the Netherlands. Results: For over 220,000 patients, a skin cancer diagnosis-related group was reimbursed in 124 healthcare centres. Conventional excision reflected $75 \%$ of treatments for skin cancer but showed large variation between practices. Large practice variation was also found for 5-fluorouracil and imiquimod creams. The practice variation of Mohs micrographic surgery and photodynamic therapy was low under the 75th percentile, but outliers at the 100th percentile were detected, which indicates that few centres performed these thera-
\end{abstract}

pies far more often than average. On average, patients received 1.8 follow-up visits in 2016. Conclusions: Claims data demonstrated large practice variation in treatments and follow-up visits of skin cancer and may be a valid and feasible data set to extract quality indicators. The next step is to investigate whether detected practice variation is unwarranted and if a reduction improves quality and efficiency of care.

(c) 2021 The Author(s)

Published by S. Karger AG, Basel

\section{Introduction}

The high incidence of skin cancer, the low mortality rate, the long lag time to recurrence and/or low rate of severe treatment-related complications make it difficult to monitor quality of skin cancer care [1]. Benchmarking is a monitoring method which originated within commercial industry and has found its way into healthcare. Originally, benchmarking was used to improve organizational issues (e.g., staffing ratios) but soon after to improve clinical outcomes by benchmarking clinical practice [2]. Benchmarking is a management approach which can be used to create a spirit of competition and to stimulate best practices at best cost [3]. A way to translate karger@karger.com www.karger.com/drm

Karger $\stackrel{\text { ' }}{5}$

GOPEN ACCESS
(C) 2021 The Author(s)

Published by S. Karger AG, Basel

This is an Open Access article licensed under the Creative Commons Attribution-NonCommercial-4.0 International License (CC BY-NC) (http://www.karger.com/Services/OpenAccessLicense), applicable to the online version of the article only. Usage and distribution for commercial purposes requires written permission.
Sven van Egmond

Department of Dermatology

Erasmus MC Cancer Institute

Dr. Molewaterplein 40, NL-3015 GD Rotterdam (The Netherlands)

s.vanegmond@erasmusmc.nl 


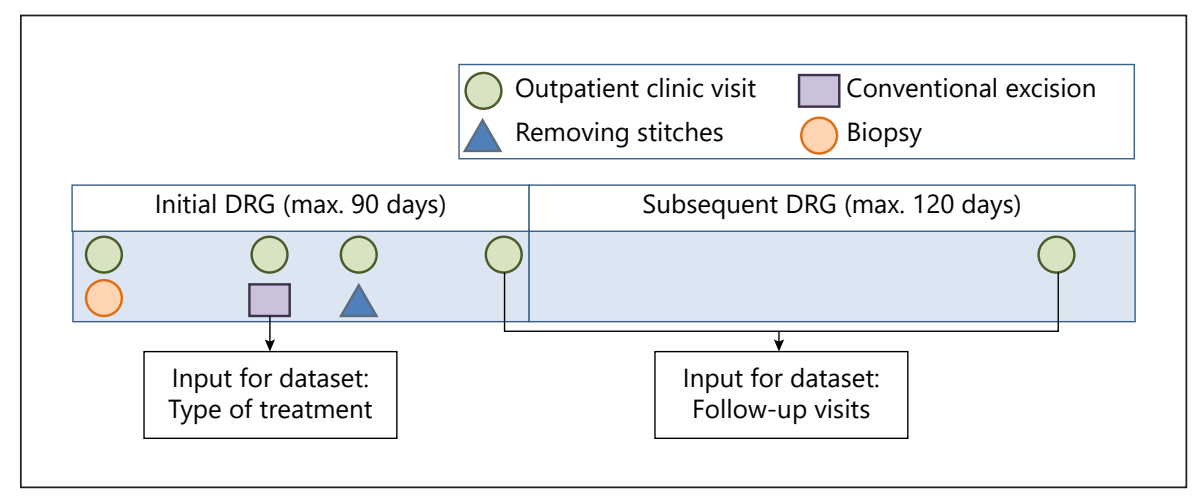

Fig. 1. Example of a diagnosis-related group (DRG) for a skin cancer patient. In this schematic example, a patient received a biopsy during the first visit, during the second visit the skin cancer was removed by conventional excision, then the sutures were removed, and thereafter, the patient received 2 follow-up visits. The conventional excision was included in the analyses of practice variation of treatments. The last 2 outpatient clinic visits, without any other registered activity on the same day, were considered follow-up visits and included in practice variation analysis of follow-up visits. benchmarking into medical care is often by using quality indicators. These quality indicators are thought to reflect quality of delivered care and can include items related to volume, complications and mortality rates [4].

Analyses of quality indicators can be used to reveal clinical practice variation, reflecting differences in care policy or outcomes between healthcare providers [5]. In only $15 \%$ of medical interventions, the choice of treatment is clear and the differences in provider judgment are negligible (e.g., hospital admission rates for hip fractures), making practice variation very common [6]. To a certain degree, practice variation is acceptable, but too much variation can be unwarranted and may be the result of under- or overtreatment $[7,8]$. In the event of undertreatment, patients may not receive the care they actually need, which reduces their chance of receiving optimal care. When overtreatment occurs, patients may be exposed to unnecessary side effects and/or costs caused by intervening more than is medically justified [9]. For example, an identical skin cancer patient may be treated by Mohs micrographic surgery (MMS) in one healthcare centre and by conventional excision in another centre.

There are sets of quality indicators which are mandatory to be registered for certain types of cancer in the Netherlands, such as complications and survival rates after resection of pancreas carcinoma and the number of incomplete resections of ovarian carcinoma [10]. However, for high-volume cancer such as skin cancer it is not feasible for healthcare providers to register quality indicators for each patient [11]. Therefore, quality indicators for skin cancer are currently only registered for stage 3C or higher melanoma in specialised melanoma centres [10]. To obtain a complete overview of skin cancer care, routine data may be a promising data tool [12].

The aim of the current study is to determine whether claims data can be used to benchmark high-volume care and to assess whether there is clinical practice variation in type of treatment and number of follow-up visits of skin cancer patients.

\section{Patients and Methods}

\section{Data Source}

Since 2005, all hospital visits and admissions in the Netherlands are categorised in diagnosis-related groups (DRGs). Each DRG includes all hospital activities and services associated with the patient care provided for a certain diagnosis. All activities related to diagnosis, treatment and follow-up are registered by the healthcare provider and included in a DRG, resulting in 1 reimbursement claim (Fig. 1) [13]. These claims are collected by healthcare insurers and subsequently sent to a national information centre (Vektis B.V., Zeist) in the Netherlands. This nationwide claims database was used for the current study. As all Dutch inhabitants are obliged to have a healthcare insurance, the coverage is over $99 \%$ and a recent study determined this database to be over $95 \%$ accurate when compared to local patient records [14].

\section{Data Extraction and Analysis}

All patients with a DRG reimbursed for a cutaneous malignancy within dermatology care in the most recent available calendar year (2016) were included. This includes patients who were diagnosed before 2016 but only had a follow-up visit in 2016 . The data sets only included cutaneous malignancies (i.e., basal cell carcinoma, squamous cell carcinoma, melanoma and rare types of skin cancer). Pre-malignancies, such as Bowen's disease and actinic 
Table 1. Total number and distribution of treatments and follow-up visits for a skin malignancy per type of treatment centre in 2016

\begin{tabular}{lcccc}
\hline $\begin{array}{l}\text { Type of healthcare } \\
\text { centre }\end{array}$ & $\begin{array}{l}\text { Number of } \\
\text { healthcare centres }\end{array}$ & $\begin{array}{l}\text { Number of } \\
\text { patients }^{\mathrm{b}}\end{array}$ & $\begin{array}{l}\text { Number of } \\
\text { follow-up visits }\end{array}$ & $\begin{array}{c}\text { Number of } \\
\text { treatments }^{\mathrm{c}}\end{array}$ \\
\hline ISTCs, $n(\%)$ & $46(37.1)$ & $24,857(11.2)$ & $55,462(14.1)$ & $17,125(12.6)$ \\
General hospitals, $n(\%)$ & $74(59.7)$ & $180,525(81.4)$ & $304,980(77.4)$ & $108,758(80.2)$ \\
University hospitals, $n(\%)$ & $8(6.5)$ & $16,498(7.4)$ & $33,530(8.5)$ & $9,715(7.2)$ \\
\hline Total & 124 & 221,880 & 393,972 & 135,598 \\
\hline
\end{tabular}

ISTCs, independent sector treatment centres. ${ }^{a}$ Healthcare centres with at least 1 patient with a follow-up visit for a skin malignancy. ${ }^{\mathrm{b}}$ Patients with at least 1 follow-up visit for a skin malignancy in $2016 .{ }^{\mathrm{c}}$ Conventional excision, Mohs micrographic surgery, photodynamic therapy, 5-fluorouracil and imiquimod cream.

keratosis, were not included. It is not possible for patients to have diagnosis codes for both a cutaneous malignancy and a pre-malignancy (e.g., squamous cell carcinoma and actinic keratosis). Unfortunately, there were no specific diagnosis codes for each subtype of skin cancer. The ICD-10 codes were introduced from 2016, which differentiates between different subtypes of skin cancer (except for basal cell carcinoma and squamous cell carcinoma), but the saturation of this data was too poor to use for the current study. Two data sets were extracted from Vektis' nationwide claims database, based on healthcare activities (Fig. 1).

One data set contained types of treatment indicated for skin cancer: conventional excision, MMS, photodynamic therapy (PDT), 5-fluorouracil and imiquimod cream. Destructive therapies, such as cryotherapy, were not included, because patients could be treated this way for their actinic keratosis during followup for their skin malignancy. If a patient received multiple treatments, e.g., topical treatment and excision, both treatments were registered. The number of treatments were stratified per healthcare centre.

The second data set contained the number of follow-up visits and was also stratified per healthcare centre. A follow-up visit was defined as a visit at the dermatology outpatient clinic after a skin cancer treatment, without any other activity registered on that day (e.g., removing sutures).

The maximum timeframe of an initial DRG is 90 days, and 120 days for a subsequent DRG. When this time limit has passed and a new care activity is registered for this patient for the same diagnosis, a subsequent DRG will be opened. The eligible DRG codes are listed in online supplementary Table S1 (for all online suppl. material, see www.karger.com/doi/10.1159/000513523). The number of referrals from other healthcare centres was determined by searching for a skin cancer DRG at another healthcare centre up to 90 days prior to the DRG in the main analysis (i.e., tertiary care). The healthcare centres were categorised as university hospital, general hospital or independent sector treatment centre and anonymised for the researchers.

The analyses were performed by using SAS software (version 9.3; SAS Institute Inc., Cary, NC, USA) and Microsoft Excel (Microsoft, Redmont, WA, USA). Charts were created of the distribution of treatment types and average number of follow-up visits per centre. Finally, after the healthcare centres were ranked according to the proportion of type of treatment or follow-up visits, percen- tiles (p0, p25, p50, p75, p100) and the differences between percentiles (p25-p75 and p0-p100) were determined to reveal practice variation. To determine whether results are skewed by small healthcare centres, a sensitivity analysis was performed by excluding the centres with the lowest quartile in terms of number of patients.

\section{Results}

In total, 124 healthcare centres in the Netherlands reimbursed at least 1 DRG for a skin malignancy within dermatology care in 2016 for over 220,000 unique patients (Table 1). The total number of patients is higher than the total number of treatments, as patients who solely received follow-up care in 2016 were included as well. Nearly 400,000 follow-up visits took place in dermatology care for skin cancer in 1 year.

\section{Treatments}

An overview of the type of treatment quality indicator scores per healthcare centre is displayed in Figure 2. In 2016, general hospitals were the most consistent in treating their skin cancer patients by conventional excision, of which the hospitals with the least conventional excisions were performing more MMS. The 2 independent sector treatment centres with the highest percentage of MMS (30 and 24\%) had a substantial proportion of their patients referred from other healthcare centres (24 and $17 \%)$. The university hospital with $33 \%$ MMS had $35 \%$ of their patients referred from other healthcare centres, compared to $2-13 \%$ referred patients of the other university hospitals.

Patients in university hospitals were treated with 5-fluorouracil or imiquimod cream in $22 \%$ of the cases, compared to 15 and $12 \%$ of patients in general hospitals and 
Table 2. Percentiles of the distribution of quality indicator scores of different types of skin malignancy treatments and follow-up visits between healthcare centres in 2016

\begin{tabular}{lcrrrrrrr}
\hline & p0 & p25 & p50 & p75 & p100 & \multicolumn{2}{c}{ Difference between } \\
\cline { 3 - 8 } & & & & & & p25-p75 & p0-p100 \\
\hline Conventional excision, \% & 33.3 & 71.3 & 77.0 & 82.4 & 90.6 & 11.1 & 57.2 \\
Mohs micrographic surgery, \% & 0 & 0.9 & 3.0 & 6.7 & 32.8 & 5.8 & 32.8 \\
5-Fluorouracil or imiquimod, \% & 0 & 10.5 & 14.6 & 18.5 & 66.7 & 7.9 & 66.7 \\
Photodynamic therapy, \% & 0 & 0.6 & 1.8 & 6.1 & 39.5 & 5.5 & 39.5 \\
Average number of follow-up visits per patient & 0.44 & 1.48 & 1.75 & 2.01 & 6.61 & 0.53 & 6.17 \\
\hline
\end{tabular}

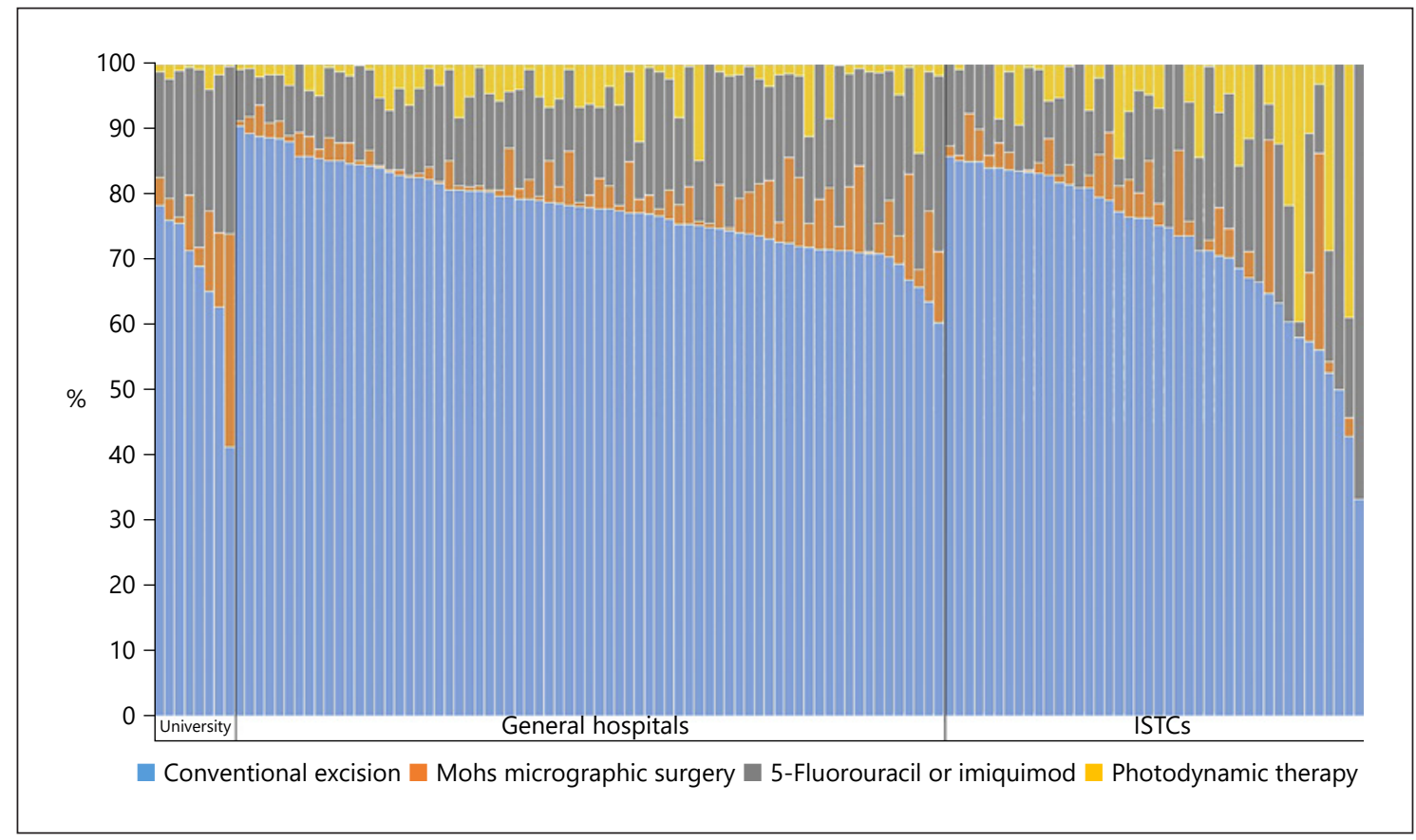

Fig. 2. Distribution of quality indicator scores of the treatments indicated for skin cancer in 2016. Each bar represents 1 healthcare centre. University, University hospitals; ISTCs, independent sector treatment centres.

independent sector treatment centres, respectively (online suppl. Fig. S1). In independent sector treatment centres, $6 \%$ of skin cancer patients were treated with PDT, while in university and general hospitals 1 and $3 \%$ of patients were treated with PDT, respectively.

On average (taking the 50th percentile), $77.0 \%$ of all malignancies were treated by conventional excision in 2016 (Table 2). This proportion was reasonably comparable with the application of conventional excision in the 25 th $(71.3 \%)$ and 75 th percentile $(82.4 \%)$. The outliers, however, also showed healthcare centres with only $33.3 \%$ (p0) or more than 90\% (p100) use of convention- al excision. Most practice variation is revealed for topical treatment for skin cancer (5-fluorouracil and imiquimod), as the p0-p100 ranges from 0 to $66.7 \%$. The low percentages of MMS and PDT until the 75th percentile $(<7 \%)$ and the high percentage at p100 (>32\%) indicate that few healthcare centres provided that care more often in 2016.

\section{Follow-up Visits}

Figure 3 provides an overview of the number of followup visits per healthcare centre. The average number of follow-up visits per patient was 2.0 for university hospi- 


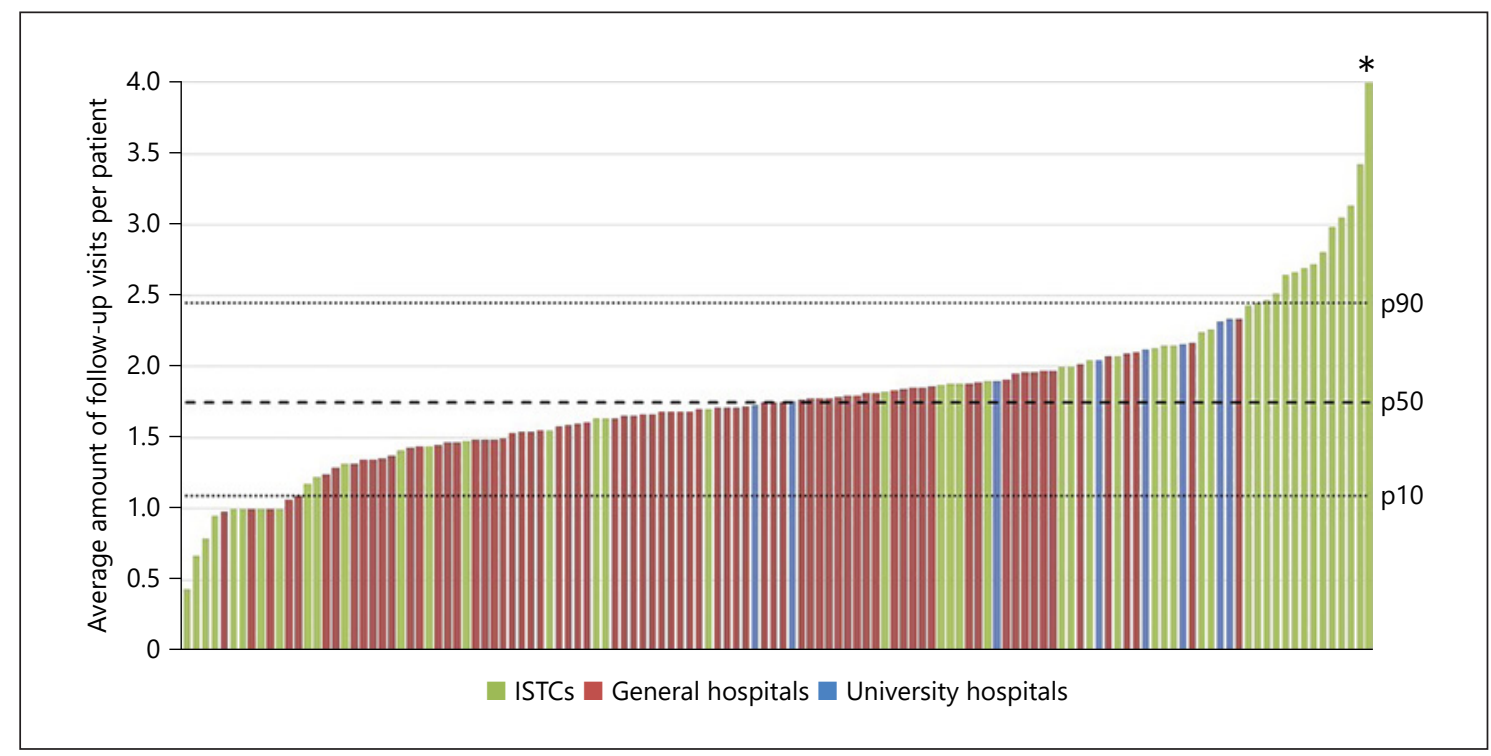

Fig. 3. Average number of follow-up visits per patient per healthcare centre in 2016. Each bar represents 1 healthcare centre. ISTCs, independent sector treatment centres. ${ }^{*}$ The $y$-axis was cut off at 4.0 for clarity; this healthcare centre's value was 6.6 .

tals, 1.7 for general hospitals and 2.0 for independent sector treatment centres. The 14 healthcare centres with the highest number of follow-up visits per patient were all independent sector treatment centres with an average of 2.4-6.6 follow-up visits per patient.

The difference in the average number of follow-up visits between healthcare centres, the 25th percentile and the 75 th percentile, was 0.53 follow-up visits per patient (Table 2). The number of follow-up visits per patient at these percentiles (1.48-2.01) did not differ much from the 50th percentile (1.75). However, the p100, showing an average number of follow-up visits of 6.61 per patient, reveals that there were some healthcare centres on the higher end contributing to practice variation.

The sensitivity analysis, which was used to detect whether results were skewed by small healthcare centres, did not differ from the main analysis concerning the interquartile range (p25-p75). The results of the sensitivity analysis were different from the main analysis on the $\mathrm{p} 0$ p100 range of topical treatments (25.2\%) and follow-up visits (2.1). This means that healthcare centres with a relatively small number of skin cancer patients deviate more from the 50th percentile and caused more practice variation than larger healthcare centres regarding these quality indicators.

\section{Discussion}

This study shows that claims data is able to detect relevant clinical practice variation in terms of skin cancer treatment and follow-up care. Proportion of specific treatments and follow-up could be valid quality indicators and routinely collected claims data may be a good data source for benchmarking.

The amount of clinical practice variation was highest for conventional excision, followed by topical creams. This variation could be explained by the referral rate of dermatologists to plastic surgeons depending on his/her surgical experience and skills, or the available facilities of the healthcare centre to provide high numbers of excisions. The practice variation in MMS and PDT was low under the 75th percentile, but outliers at the 100th percentile were detected. MMS and PDT are treatments which were (and are) not provided in all healthcare centres, which means that there has to be practice variation. As shown by Arits et al. [15], PDT is both more expensive and less effective than 5-fluorouracil and imiquimod cream, which led to guideline changes in 2014 [16]. The high amount of PDT in some healthcare centres might be explained by lack of knowledge of the guideline change. The near $100 \%$ compliance rate of conventional PDT might be a rationale to prefer this treatment for a subset of patients of whom it is to be expected that they will not 
comply with creams at home (e.g., stopping treatment too early due to side effects). However, it may also have been stimulated by a financial incentive, as PDT is more profitable for healthcare centres than conventional excision and topical treatments.

The average amount of follow-up visits per skin cancer patient was 1.8 in 2016. Considering that skin cancer patients comprise $24 \%$ of all dermatology patients, these follow-up visits account for a large part of dermatology care [17]. Comparing the 25 th percentile to the 75 th percentile indicates little practice variation between healthcare centres regarding the number of follow-up visits per skin cancer patient. However, it is remarkable that the 14 healthcare centres with the highest number of follow-up visits per patient were all independent sector treatment centres.

Making use of claims data has some limitations. As the information was aggregated, it should be interpreted carefully. It does not allow analyses on absolute frequencies, but rather a comparison of relative frequencies between healthcare providers. No conclusions regarding under- or overtreatment can be drawn on the basis of practice variation found in the current study, because centres could treat different patient populations. For instance, due to lack of detailed information on the patient level (e.g., age, type of tumour), the case mix of each centre could not be determined. For this reason, it was not possible for the authors to determine whether the high percentage of MMS and high number of follow-up visits are due to specialisation in complex skin cancer care. Although the number of referrals provides an indication, no causality can be established. Strengths of claims databases are that it is routinely registered data, it is virtually complete due to obligatory registration and that the summaries of quality indicators of claims data match summaries of quality indicators of the actual medical records [18].

The next step is to determine whether the practice variation found in our study is warranted. Institutions such as the Ministry of Health or health insurers (in collaboration with clinical experts) could request healthcare centres to retrieve their own quality indicator scores from Vektis and investigate why certain centres deviate from the average. This process of audit and feedback might already effectively reduce possible unwarranted practice variation [19]. There are several other options to reduce the variation, such as the development and implementation of guidelines (most common strategy), improving shared decision-making and introduction of financial incentives [20-25]. Multifaceted strategies have been prov- en to be more effective in reducing practice variation than single strategies [26].

In conclusion, claims data can be used to benchmark high-volume care and to reveal clinical practice variation on routinely collected quality indicators. The current study revealed that there might be under- and/or overtreatment in the case of conventional excisions and topical creams. In addition, it showed that there is little practice variation regarding follow-up visits, but it was surprising to see that the 14 healthcare centres with the highest number of follow-up visits per patient were all independent sector treatment centres. It should be explored if the variation found in the current study is warranted and if further actions should be undertaken to reduce the practice variation.

\section{Key Message}

Analysis of claims data revealed large practice variation in skin cancer treatments and follow-up care

\section{Statement of Ethics}

The authors have no ethical conflicts to disclose. The current study was approved by "Zorgverzekeraars Nederland," an umbrella organization of 10 health insurers in the Netherlands who are responsible for the patient records in the Vektis database."

\section{Conflict of Interest Statement}

The authors have no conflicts of interest to declare.

\section{Funding Sources}

This project was funded by Citrienfund (Dutch Ministry of Health, Welfare and Sport). The funder had no involvement in the study design; in the collection, analysis and interpretation of data; in the writing of the report; and in the decision to submit the article for publication.

\section{Author Contributions}

All authors have participated in the concept and design, analysis and interpretation of data, drafting or revising of the manuscript, and they approved the paper as submitted. 


\section{References}

1 Verkouteren JA, Ramdas KH, Wakkee M, Nijsten T. Epidemiology of basal cell carcinoma: scholarly review. Br J Dermatol. 2017 Aug; 177(2):359-72.

2 Ellis J. Sharing the evidence: clinical practice benchmarking to improve continuously the quality of care. J Adv Nurs. 2000 Jul;32(1): 215-25.

3 Ettorchi-Tardy A, Levif M, Michel P. Benchmarking: a method for continuous quality improvement in health. Healthc Policy. 2012 May;7(4):e101-19.

4 Lind S, Adolfsson J, Axelsson B, Fürst CJ. Quality indicators for palliative and end of life care: a review of Swedish policy documents. BMJ Support Palliat Care. 2015 Dec;5(4): 413-9.

5 Corallo AN, Croxford R, Goodman DC, Bryan EL, Srivastava D, Stukel TA. A systematic review of medical practice variation in OECD countries. Health Policy. 2014 Jan;114(1):514.

6 Goodwin JS. Tracking Medicine: A Researcher's Quest to Understand Health Care By John E. Wennberg. Am J Epidemiol. 2011;174(2): 252.

7 Wennberg JE. Unwarranted variations in healthcare delivery: implications for academic medical centres. BMJ. 2002 Oct;325(7370): 961-4.

8 OECD. Geographic variations in health care: what do we know and what can be done to improve health system performance? OECD health policy studies. 2014.

9 Vektis. Rapportage indicatoren indicatiestelling (praktijkvariatie). 2011.

10 (DICA) DIFCA. Jaarrapportage 2018 - Oncologische registraties. 2018.
11 Dewi S. Effective excellence in nursing: Bridging the gap between measurement of quality of nursing care and clinical reality. Utrecht: Universiteit van Utrecht; 2016.

12 Viboud C, Charu V, Olson D, Ballesteros S, Gog J, Khan F, et al. Demonstrating the use of high-volume electronic medical claims data to monitor local and regional influenza activity in the US. PLoS One.2014 Jul;9(7):e102429.

13 van de Ven WP, Schut FT. Universal mandatory health insurance in the Netherlands: a model for the United States? Health Aff (Millwood). 2008 May-Jun;27(3):771-81.

14 Eindhoven DC, van Staveren LN, van Erkelens JA, Ikkersheim DE, Cannegieter SC, Umans VA, et al. Nationwide claims data validated for quality assessments in acute myocardial infarction in the Netherlands. Neth Heart J. 2018 Jan;26(1):13-20.

15 Arits AH, Spoorenberg E, Mosterd K, Nelemans P, Kelleners-Smeets NW, Essers BA. Cost-effectiveness of topical imiquimod and fluorouracil vs. photodynamic therapy for treatment of superficial basal-cell carcinoma. Br J Dermatol. 2014 Dec;171(6):1501-7.

16 Nederlandse Vereniging voor Dermatologie en Venereologie (NVDV). Evidence-based Richtlijn Basaalcelcarcinoom. Utrecht, the Netherlands: NVDV; 2014.

17 Open data van de Nederlandse Zorgautoriteit. [cited 2020 Apr 6]. Available from: https:// www.opendisdata.nl/

18 MacLean CH, Louie R, Shekelle PG, Roth CP, Saliba D, Higashi T, et al. Comparison of administrative data and medical records to measure the quality of medical care provided to vulnerable older patients. Med Care. 2006 Feb;44(2):141-8.
19 Ivers N, Jamtvedt G, Flottorp S, Young JM, Odgaard-Jensen J, French SD, et al. Audit and feedback: effects on professional practice and healthcare outcomes. Cochrane Database Syst Rev. 2012 Jun;(6):CD000259.

20 Tomson CR, van der Veer SN. Learning from practice variation to improve the quality of care. Clin Med (Lond). 2013 Feb;13(1):19-23.

21 Brabers AE, van Dijk L, Groenewegen PP, van Peperstraten AM, de Jong JD. Does a strategy to promote shared decision-making reduce medical practice variation in the choice of either single or double embryo transfer after in vitro fertilisation? A secondary analysis of a randomised controlled trial. BMJ Open. 2016 May;6(5):e010894.

22 Westert GP, Faber M. Commentary: the Dutch approach to unwarranted medical practice variation. BMJ. 2011 Mar;342:d1429.

23 Kennedy PJ, Leathley CM, Hughes CF. Clinical practice variation. Med J Aust. 2010 Oct; 193(S8):S97-9.

24 Baiardini I, Braido F, Bonini M, Compalati E, Canonica GW. Why do doctors and patients not follow guidelines? Curr Opin Allergy Clin Immunol. 2009 Jun;9(3):228-33.

25 Lugtenberg M, Zegers-van Schaick JM, Westert GP, Burgers JS. Why don't physicians adhere to guideline recommendations in practice? An analysis of barriers among Dutch general practitioners. Implement Sci. 2009 Aug;4(1):54.

26 van der Veer SN, Jager KJ, Nache AM, Richardson D, Hegarty J, Couchoud C, et al. Translating knowledge on best practice into improving quality of RRT care: a systematic review of implementation strategies. Kidney Int. 2011 Nov;80(10):1021-34. 